\section{Recognition of hemophagocytic lymphohistiocytosis in sickle cell vaso-occlusive crises is a potentially lifesaving diagnosis}

Hemophagocytic lymphohistiocytosis (HLH) is a rare but aggressive and potentially life-threatening syndrome caused by a hyperinflammatory response leading to organ damage. ${ }^{1,2}$ It is characterized by fevers, cytopenias, hepatosplenomegaly, hyperferritinemia, hypertriglyceridemia and hemophagocytosis by activated macrophages. ${ }^{3} \mathrm{HLH}$ can either be primary (inherited) or secondary. Primary HLH generally presents in infancy and is associated with mutations that affect cytotoxic Tcell or inflammasome function. ${ }^{4,5}$ Secondary HLH is thought to be more common and to be due to infection, hematologic malignancy, autoimmune disorders or drugs. ${ }^{1}$

Sickle cell anemia is caused by homozygous mutations of the beta $(\beta)$-globin gene in which the sixth amino acid, glutamic acid, is replaced by valine, producing a hydrophobic motif in deoxygenated hemoglobin $S$ (HbS). ${ }^{6}$ Patients with sickle cell anemia suffer from complications of chronic hemolysis and recurrent vaso-occlusive crises (VOC). ${ }^{7}$ Patients who are compound heterozygotes for $\mathrm{HbS}$ and hemoglobin $\mathrm{C}$ also can suffer from VOC. HLH in sickle cell disease and related hemoglobinopathies has been described but is not well characterized and has been associated with mycobacterium avium-complex infection, malaria, transfusion and bone pain crises..$^{8-11} \mathrm{HLH}$ in sickle cell patients is under-recognized so the true incidence is not known. HLH is a fatal syndrome that requires a timely diagnosis in order to initiate life-saving therapy. The clinical and laboratory features of sickle cell VOC and HLH partially overlap, making the diagnosis of HLH difficult in these patients. Here we present 2 cases of HLH in sickle cell patients where timely diagnosis and treatment ultimately led to favorable outcomes.
Patient 1 is a 35 -year old African American female with a history of sickle cell anemia, chronic lower extremity ulcerations, prior admissions for acute chest syndrome, asthma and migraine who presented to our hospital with worsening of chronic lower extremity pain. Her lifetime transfusion history was approximately 23 units of red cells and her baseline ferritin was 442 $\mathrm{ng} / \mathrm{mL}$. She was admitted for treatment of presumptive VOC. While in hospital, she developed recurrent fevers that did not respond to broad-spectrum intravenous (IV) antibiotics. Blood cultures and work-up for infection were unrevealing. The patient deteriorated clinically and was transferred to the intensive care unit. She developed hypoxia and imaging showed diffuse lymphadenopathy. During her hospital stay she developed worsening transaminitis [alanine transaminase (ALT) and aspartate aminotransferase (AST)], coagulopathy, pancytopenia and acute kidney injury (AKI) requiring hemodialysis. She was transfused four units of packed red blood cells and underwent an exchange transfusion that did not change her clinical status. Human immunodeficiency virus (HIV), hepatitis $\mathrm{A} / \mathrm{B} / \mathrm{C}$, cytomegalovirus (CMV) and parvovirus B19 serological tests were negative. Although initially negative for acute Epstein-Barr virus (EBV) infection, she ultimately had a mild transient viremia (263 copies/mL; normal < 200).

Laboratory testing demonstrated hyperferritinemia (9597 ng/mL; normal 10-109), hypertriglyceridemia (340 $\mathrm{mg} / \mathrm{dL}$; normal 40-200), hypofibrinogenemia $(70 \mathrm{mg} / \mathrm{dL}$; normal 180-460), and an elevated soluble IL-2R (5250 $\mathrm{pg} / \mathrm{mL}$; normal 190-570). A bone marrow (BM) biopsy showed hemophagocytosis, histiocytosis and BM damage (Figure 1A). Based on these clinical parameters and pathological finding, a diagnosis of HLH was made. BM cultures and acid-fast bacilli stains were negative for bacteria, fungi and mycobacteria. Approximately one month after hospital admission and three weeks after the completion of the exchange transfusion, the patient was started on intravenous immunoglobulin $(400 \mathrm{mg} / \mathrm{kg}$

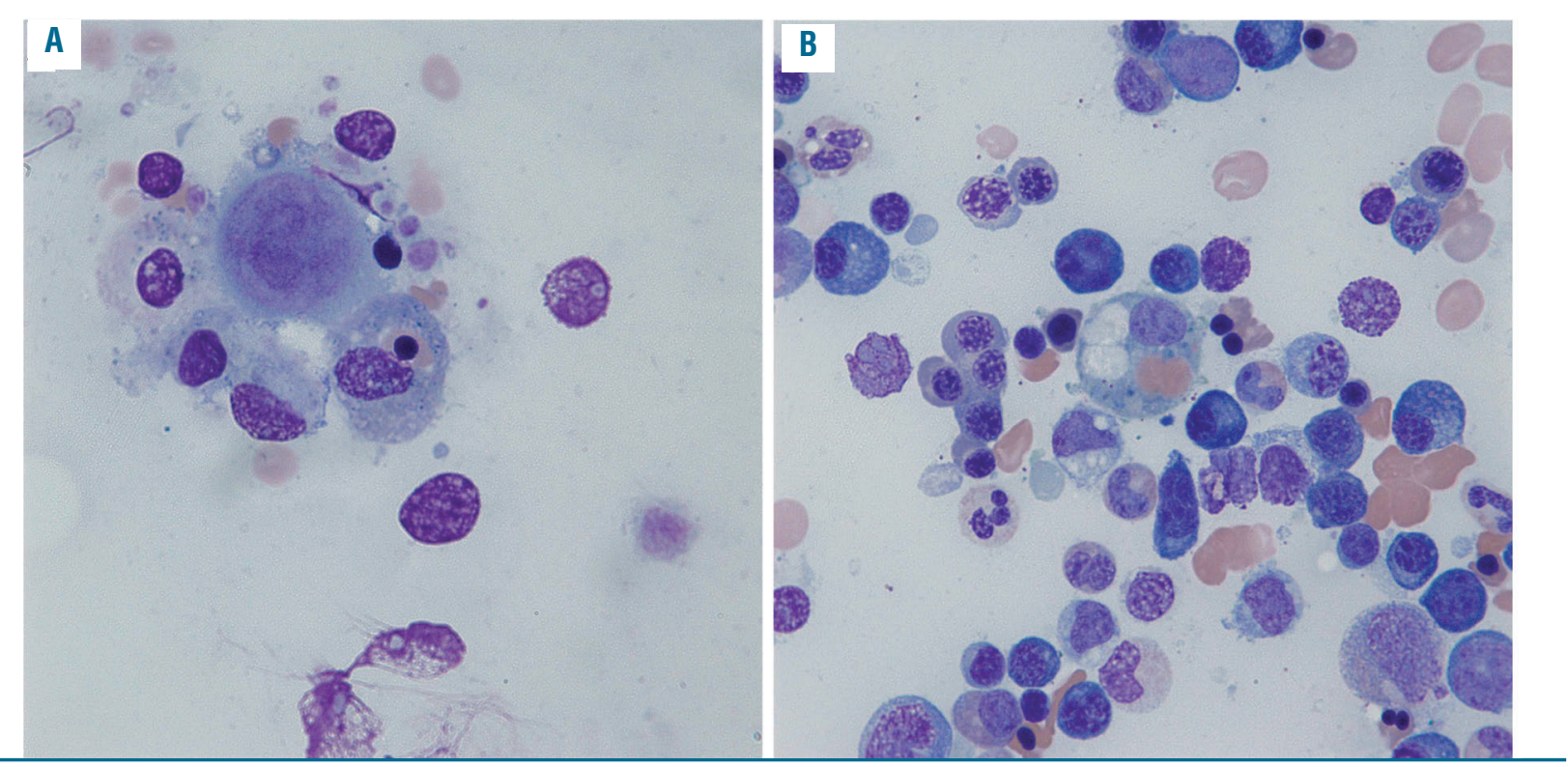

Figure 1. Wright-Giemsa staining of bone marrow (BM) aspirate showing hemophagocytosis. (A) Wright-Giemsa stained BM aspirate smear at $1000 x$ of Patient 1 showing a histiocyte phagocytosing a nucleated red blood cell (RBC). (B) Wright-Giemsa stained BM aspirate smear at $1000 x$ of Patient 2 showing a histiocyte phagocytosing two RBCs with a mild erythroid hyperplasia and mild reactive plasmacytosis. 
A

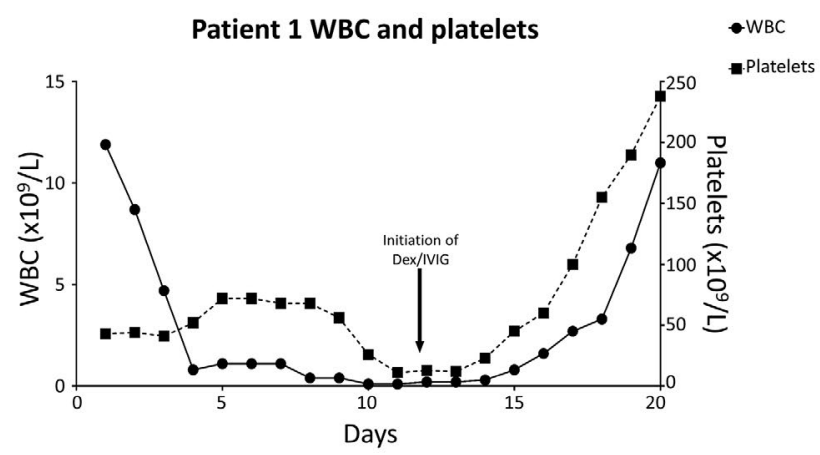

B

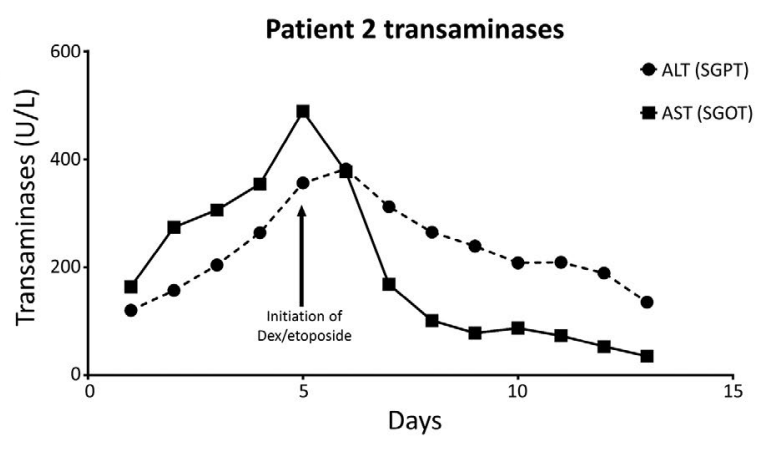

Figure 2. Patients showed improvement in blood markers of end-organ damage after initiation of immunosuppressive therapy. (A) Graphic trend of the white blood cell (WBC) count and platelet count of Patient 1 showing recovery of WBC and platelets after initiation of dexamethasone (Dex) and intravenous immunoglobulin (IVIG) treatment for hemophagocytic lymphohistiocytosis (HLH) (black arrows). (B) Graphic trend of transaminases in U/L of Patient 2 showing a reduction in transaminitis of both alanine transaminase (ALT) and aspartate aminotransferase (AST) following initiation of Dex and etoposide treatment for HLH.

daily) and high-dose dexamethasone (Dex) (40 mg daily) for five days with dramatic resolution of fevers, leukopenia, thrombocytopenia, transaminitis, diffuse lymphadenopathy and hemodialysis dependence (Figure 2A). She was subsequently discharged to a rehabilitation center and from there ultimately went home.

Patient 2 is a 56-year old male with a history of hemoglobin SC (HbSC) disease who presented with sickle cell VOC and a fever of $101.2^{\circ} \mathrm{F}$. His lifetime transfusion history was 82 units of red cells and his baseline ferritin was $199 \mathrm{ng} / \mathrm{mL}$. Initially, the patient had a mild AKI, liver function abnormalities (ALT/AST 112/101 U/L) and coagulopathy. A work-up for infection demonstrated mild CMV viremia (viral load 2500) but otherwise no evident source of infection, with blood cultures that were negative and complete blood count that was unremarkable. Computed tomography scan of chest, abdomen and pelvis was performed and this did not suggest any focus of infection. Despite broad-spectrum antibiotics and transfusion of a total of four units of red cells, the patient's AKI and transaminitis worsened, fevers continued, and his mental status deteriorated. Laboratory studies demonstrated an elevated ferritin to $>4787 \mathrm{ng} / \mathrm{mL}$ (normal 10-109) and soluble IL-2R of $4410 \mathrm{pg} / \mathrm{mL}$ (normal 190-570). HLH was suspected and the patient underwent a BM biopsy that showed histiocytosis with hemophagocytosis (Figure 1B). The patient was started on Dex (20 mg daily) and etoposide $\left(75 \mathrm{mg} / \mathrm{m}^{2}\right)$ with rapid resolution of his altered mentation, liver function, coagulopathy, AKI and fevers (Figure 2B).

Both sickle cell VOC and HLH can induce multi-organ failure and be fatal. ${ }^{12}$ As treatment is markedly different for these disorders, consideration of HLH in a severely ill sickle cell patient who is not responding to standard supportive measures is critically important to allow the initiation of potentially life-saving therapy..$^{12}$ It is important to highlight that the diagnosis of HLH in the sickle cell patient population is challenging, and that hyperferritinemia and hemophagocytosis alone are not sufficient to support a diagnosis of HLH in acutely ill sickle cell patients. Sickle cell patients may have hyperferritinemia due to iron load from multiple transfusions compounded by acute inflammatory illnesses. Hemophagocytosis is a well-documented occurrence during VOCs and concomitant viral infections, and treatment-related complications such as delayed transfusion reactions can precipitate multi-organ failure and inflammatory biology that mimic HLH.

Our patients had fever, pancytopenia, hypertriglyceridemia, elevated ferritin greater than $500 \mathrm{ng} / \mathrm{mL}$, hemophagocytosis in BM, and elevated soluble IL-2R, which fulfill 6 of the 8 diagnostic criteria of the HLH-2004 trial. ${ }^{13}$ Their clinical courses did not suggest delayed transfusion reaction and despite a concerted effort, no specific alternative diagnosis was found to explain their fevers or progressive multisystem organ dysfunction. Furthermore, our patients' rapid and dramatic resolution of symptoms and multi-organ failure following initiation of immunosuppressive therapy indicates that it is likely that HLH was the correct diagnosis in both cases. The cause of $\mathrm{HLH}$ in our patients is unknown. Patient 1 did have a transient EBV viremia, and EBV reactivation is a relatively common cause of secondary HLH. ${ }^{1,14}$ Patient 2 had a transient CMV viremia, which has also been associated with HLH..$^{15}$ Nonetheless, the level of viremia was modest in both cases, and it remains possible that the sickle cell VOC itself played an important role in precipitating the HLH episode.

Although the literature of patients with sickle cell disease and HLH is sparse, most cases have been associated with infection. ${ }^{11,16}$ Most cases of HLH in sickle cell disease in the literature were treated with antibiotics or supportive care; corticosteroids and an interleukin-1 receptor antagonist were used to treat one patient who succumbed to complications of HLH. ${ }^{9-11,16}$ While treatment for HLH has not been adequately studied in randomizedcontrolled trials due to its rarity, immunosuppression with corticosteroids, IV immunoglobulin, and etoposide has been effective either alone or in combination. ${ }^{1}$ Our cases illustrate the importance of having a high suspicion for HLH in patients with sickle cell anemia with fever, pancytopenia, and multi-organ dysfunction that do not respond to therapy, as timely treatment of HLH can result in rapid and profound clinical improvement.

Orly Leiva, ${ }^{1,2}$ Lillian McMahon, ${ }^{1}$ J. Mark Sloan, John Lee ${ }^{3}$ and Adam Lerner'

'Department of Medicine, Boston University School of Medicine; ${ }^{2}$ Department of Medicine, Brigham and Women's Hospital, Boston and ${ }^{3}$ Department of Pathology \& Laboratory Medicine, Boston University School of Medicine, MA, USA 
Acknowledgments: we would like to acknowledge and thank Dr. Martin Steinberg for his expertise and insights in writing this manuscript. We would also like to thank the patients for teaching us something new every day and the entire medical team who took care of them.

Correspondence: ADAM LERNER - adam.lerner@bmc.org doi:10.3324/haematol.2018.206458

Information on authorship, contributions, and financial \& other disclosures was provided by the authors and is available with the online version of this article at www. haematologica.org.

\section{References}

1. Ramos-Casals M, Brito-Zeron P, Lopez-Guillermo A, Khamashta MA, Bosch X. Adult haemophagocytic syndrome. Lancet. 2014;383(9927):1503-1516.

2. Schram AM, Berliner N. How I treat hemophagocytic lymphohistiocytosis in the adult patient. Blood. 2015;125(19):2908-2914.

3. Janka GE, Lehmberg K. Hemophagocytic syndromes--an update. Blood Rev. 2014;28(4):135-142.

4. Pachlopnik Schmid J, Cote M, Menager MM, et al. Inherited defects in lymphocyte cytotoxic activity. Immunol Rev. 2010;235(1):10-23.

5. Canna SW, de Jesus AA, Gouni S, et al. An activating NLRC4 inflammasome mutation causes autoinflammation with recurrent macrophage activation syndrome. Nat Genet. 2014;46(10):11401146.

6. Piel FB, Steinberg MH, Rees DC. Sickle Cell Disease. N Engl J Med. 2017;376(16):1561-1573.
7. Rees DC, Williams TN, Gladwin MT. Sickle-cell disease. Lancet. 2010;376(9757):2018-2031.

8. Thung I, Broome HE. Hemophagocytosis in a patient with sickle cell disease. Blood. 2016;127(3):369.

9. Rehman JU, Bhabri N, Waleed A, Maulawi A, Aslam M. Falciparum malaria in a patient with sickle cell trait with hemophagocytosis and secondary pancytopenia. Ann Hematol. 2012;91(8):1329-1330.

10. Kio E, Onitilo A, Lazarchick J, Hanna M, Brunson C, Chaudhary U. Sickle cell crisis associated with hemophagocytic lymphohistiocytosis. Am J Hematol. 2004;77(3):229-232.

11. Chamsi-Pasha MA, Alraies MC, Alraiyes AH, Hsi ED. Mycobacterium avium Complex-Associated Hemophagocytic Lymphohistiocytosis in a Sickle Cell Patient: An Unusual Fatal Association. Case Rep Hematol. 2013;2013:291518.

12. Hassell KL, Eckman JR, Lane PA. Acute multiorgan failure syndrome: a potentially catastrophic complication of severe sickle cell pain episodes. Am J Med. 1994;96(2):155-162.

13. Henter JI, Horne A, Arico M, et al. HLH-2004: Diagnostic and therapeutic guidelines for hemophagocytic lymphohistiocytosis. Pediatr Blood Cancer. 2007;48(2):124-131.

14. Filipovich A, McClain K, Grom A. Histiocytic disorders: recent insights into pathophysiology and practical guidelines. Biol Blood Marrow Transplant. 2010;16(1 Suppl):S82-89.

15. Cattaneo C, Oberti M, Skert C, et al. Adult onset hemophagocytic lymphohistiocytosis prognosis is affected by underlying disease and coexisting viral infection: analysis of a single institution series of 35 patients. Hematol Oncol. 2017;35(4):828-834.

16. Kashif M, Tariq H, Ijaz M, Gomez-Marquez J. Disseminated Histoplasmosis and Secondary Hemophagocytic Syndrome in a Non-HIV Patient. Case Rep Crit Care. 2015;2015:295735. 